\title{
Epidemiological, clinical and biochemical characterization of the p.(Ala359Asp) SMPD1 variant causing Niemann-Pick disease type B
}

\author{
Mariana Acuña ${ }^{1,2}$, Pablo Martínez ${ }^{1,2}$, Carol Moraga ${ }^{2,3}$, Xingxuan $\mathrm{He}^{4}$, Mauricio Moraga ${ }^{5}$, Bessie Hunter ${ }^{6}$, \\ Peter Nuernberg ${ }^{7}$, Rodrigo A Gutiérrez ${ }^{2,3}$, Mauricio González ${ }^{2,8}$, Edward H Schuchman ${ }^{4}$, José Luis Santos ${ }^{9}$, \\ Juan Francisco Miquel ${ }^{1,2}$, Paulina Mabe ${ }^{\star, 10}$ and Silvana Zanlungo ${ }^{\star, 1,2}$
}

\begin{abstract}
Niemann-Pick disease type B (NPDB) is a rare, inherited lysosomal storage disorder that occurs due to variants in the sphingomyelin phosphodiesterase 1 (SMPD1) gene and the resultant deficiency of acid sphingomyelinase (ASM) activity. While numerous variants causing NPDB have been described, only a small number have been studied in any detail. Herein, we describe the frequency of the p.(Ala359Asp) variant in the healthy Chilean population, and determine the haplotype background of homozygous patients to establish if this variant originated from a common founder. Genomic DNA samples from 1691 healthy individuals were analyzed for the p.(Ala359Asp) variant. The frequency of p.(Ala359Asp) was found to be 1/105.7, predicting a disease incidence of $1 / 44960$ in Chile, higher than the incidence estimated by the number of confirmed NPDB cases. We also describe the clinical characteristics of 13 patients homozygous for p.(Ala359Asp) and all of them had moderate to severe NPDB disease. In addition, a conserved haplotype and shared $280 \mathrm{~Kb}$ region around the SMPD1 gene was observed in the patients analyzed, indicating that the variant originated from a common ancestor. The haplotype frequency and mitochondrial DNA analysis suggest an Amerindian origin for the variant. To assess the effect of the p.(Ala359Asp) variant, we transfected cells with the ASM-p.(Ala359Asp) cDNA and the activity was only $4.2 \%$ compared with the wild-type cDNA, definitively demonstrating the causative effect of the variant on ASM function. Information on common variants such as p.(Ala359Asp) is essential to guide the successful implementation for future therapies and benefit to patients.
\end{abstract}

European Journal of Human Genetics (2016) 24, 208-213; doi:10.1038/ejhg.2015.89; published online 29 April 2015

\section{INTRODUCTION}

Acid sphingomyelinase (ASM) is a lysosomal hydrolase that catalyzes the breakdown of sphingomyelin into ceramide and phosphocholine. It is encoded by the sphingomyelin phosphodiesterase 1 (SMPD1, gene ID 6609, MIM\# 607608, GenBank\# M81780.1) gene located on chromosome 11 and is comprised of $4585 \mathrm{bp}$ of length distributed in six exons. ${ }^{1}$ Variants in this gene are responsible for Niemann-Pick disease types A and B (NPDA and NPDB, respectively). ${ }^{2,3}$ Patients with NPDA generally have lower ASM activity than those with NPDB and display a severe phenotype with an early involvement of the central nervous system, while NPDB patients mostly exhibit visceral involvement. ${ }^{2,4,5}$ Patients with intermediate phenotypes between types A and B NPD have also been described. ${ }^{6,7}$ These patients represent the expected continuum caused by inheriting different variants in the ASM gene. ${ }^{2}$ More than 100 pathogenic variants in the SMPD1 gene have been identified throughout the gene, albeit most are located in exon $2 .{ }^{8}$

The clinical signs that characterize NPDB are moderate to severe hepatosplenomegaly, pancytopenia, hypercholesterolemia, hypertriglyceridemia and progressive interstitial lung damage. NPDB disease severely affects quality-of-life and may produce death from respiratory or liver failure during the first three decades of life. ${ }^{5,9}$ To date there is no specific treatment to prevent disease progression, although early detection improves prognosis through timely management of complications. Currently, clinical trials are being conducted to evaluate the safety and efficacy of enzyme replacement therapy for NPDB through infusion of recombinant ASM. ${ }^{10}$ The experience provided by enzyme replacement therapy in other lysosomal diseases suggests that this therapeutic approach should be very effective in NPDB. ${ }^{10,11}$

NPDA and NPDB are panethnic, but it is well-documented that type A-causing SMPD1 variants occur more frequently among individuals of Ashkenazi Jewish ancestry than in the general population. ${ }^{3}$ Three common variants associated with Ashkenazi Jewish individuals have been described: p.R496L, p.L302P and p.P330fs. Based on this genetic information, preconception genetic screening for these variants has been undertaken within the Ashkenazi Jewish population, and the

${ }^{1}$ Departamento de Gastroenterología, Facultad de Medicina, Pontificia Universidad Católica de Chile, Santiago, Chile; ${ }^{2}$ FONDAP Center for Genome Regulation (CGR), Santiago, Chile; ${ }^{3}$ Departamento de Genética Molecular y Microbiología, Facultad de Ciencias Biológicas, Pontificia Universidad Católica de Chile, Santiago, Chile; ${ }^{4}$ Department of Genetics \& Genomic Sciences, Icahn School of Medicine at Mount Sinai, New York, NY, USA; ${ }^{5}$ Programa de Genética Humana, Instituto de Ciencias Biomédicas, Facultad de Medicina, Universidad de Chile, Santiago, Chile; ${ }^{6}$ Hospital Luis Calvo Mackenna, Santiago, Chile; ${ }^{7}$ Cologne Center for Genomics (CCG), University of Cologne, Köln, Germany; ${ }^{8}$ INTA, Universidad de Chile, Santiago, Chile; ${ }^{9}$ Departamento de Nutrición, Pontificia Universidad Católica de Chile, Santiago, Chile; ${ }^{10}$ Hospital Dr. Exequiel González Cortés, Unidad de Neurología, Santiago, Chile

*Correspondence: Dr P Mabe, Hospital Dr. Exequiel González Cortés, Unidad de Neurología, Ramón Barros Luco 3301, San Miguel, Santiago, Chile. Tel: +56 225765898 ; Fax: +56 22294 1254; E-mail: pymabe@yahoo.de

or Professor S Zanlungo, Departamento de Gastroenterología, Facultad de Medicina, Pontificia Universidad Católica de Chile, Marcoleta 367, Casilla 114-D, Santiago, Chile. Tel: +56 22354 3833; Fax: +56 22639 7780; E-mail: silvana.zanlungo@gmail.com

Received 24 November 2014; revised 19 March 2015; accepted 25 March 2015; published online 29 April 2015 
frequency of NPDA infants within this group has dropped significantly. Several SMPD1 variants causing NPDB occur frequently in other populations, including p.R608del, found with increased frequency in patients from North Africa; p.H421Y, present in the Saudi Arabian population; and the p.W391G variant common in the Gypsy population, among others. ${ }^{2,12-15}$ The p.(Ala359Asp) variant, was initially described as p.A357D using the historical nomenclature by Simonaro et $a l,{ }^{8}$ and this is the only published study of this variant. Indeed, the two patients included in this study were Chilean and as far as we know this variant has been found only in Chilean patients.

Currently, the true incidence of NPDB in many countries remains unknown, as the treating clinicians often under diagnose these diseases. In Chile, there are at least 45 confirmed NPDB cases, of which 41 are unrelated (Mabe, unpublished data). DNA sequencing has been carried out in 22 Chilean patients, 21 of them unrelated. Nineteen of these were homozygous for the p.(Ala359Asp) variant and three were compound heterozygotes (Mabe and Zanlungo, unpublished results). Considering the high frequency of the p.(Ala359Asp) variant in NPDB Chilean patients, we postulated that this variant originated from a single common ancestor that spread the variant across generations in the Chilean population, rather than emerging from recurrent mutagenic events.

The present study characterizes the epidemiological and molecular properties of the p.(Ala359Asp) variant and collects phenotypic information on homozygous patients. We analyzed the frequency of the p.(Ala359Asp) variant in a representative cohort of the general healthy Chilean population, and used this information to estimate the theoretical incidence rate of this disease in Chile. In six homozygous patients, we evaluated homozygosity of genetic markers around the p.(Ala359Asp) SMPD1 region that would predict a common ancestor. We conducted haplotype frequency and mitochondrial DNA (mtDNA) analysis to determine the possible origin of this ancestor. Furthermore, we evaluated the effect of the p.(Ala359Asp) variant on ASM activity to prove that this amino acid substitution has a direct causative effect on ASM function. The results are likely to have an important impact on the implementation of enzyme replacement therapy for NPDB in Chile, and provide some of the most extensive epidemiological data on any SMPD1 variant to date.

\section{MATERIALS AND METHODS}

Two nomenclatures have been used for describing the variants in NPDB; the historical one, which refers to a reference sequence that has two codons less due to a naturally occurring length polymorphism in the portion of the SMPD1 gene encoding the signal peptide, and the current one that includes these two codons. In this paper the p.(Ala359Asp) variant was named using the current gene sequence (RefSeq NM_000543.3, c.1076C > A), but the historical nomenclature has been used for all the other variants to facilitate reading for clinicians and more generally investigators familiar with NPDB.

\section{DNA samples}

We studied an urban cohort of 1921 DNA samples extracted from the blood of non-related individuals from Santiago city. They were of both sexes, between 20-85 years old, and randomly selected from a population in the southeastern urban area of Santiago.

To study genetic markers and mtDNA, we analyzed DNA samples from six unrelated NPDB patients homozygous for the p.(Ala359Asp) variant.

All procedures followed were in accordance with the ethical standards of the ethics board from the Faculty of Medicine, Pontificia Universidad Católica de Chile. Informed consent was obtained from all patients for being included in the study.

\section{Taqman allelic discrimination assay}

The analysis of the SMPD1 p.(Ala359Asp) variant was performed by Taqman allelic discrimination using the TaqMan Genotyping Master Mix (Applied Biosystems, Foster City, CA, USA) as detailed in the Supplementary Materials and Methods. DNA samples testing positive for the p.(Ala359Asp) variant were confirmed by sequencing.

\section{Clinical characteristics of NPDB in homozygous p.(Ala359Asp) patients}

Analysis included data of 13 female $(n=9)$ and male $(n=4)$ Chilean patients with NPDB who were homozygous for the p.(Ala359Asp) variant. These patients belonged to 12 apparently unrelated families. The mean ASM activity was measured in fibroblasts, white blood cells and in dried-blood filter paper using different techniques. The mean activity was $4.8 \pm 3.2 \%$ (range $0-10.7 \%$ ) of normal values (referred as the median of the normal value given by each laboratory).

Clinical anamnesis and physical examination including anthropometric evaluation, a complete neurological assessment, eye fundoscopy and laboratory analysis (detailed in the Supplementary Materials and Methods) were performed for each patient. Pulmonary function was evaluated by standard spirometry and by pulse oximetry, both at rest and during a five-min walk, in 12 patients over the age of 5 .

Common ancestor haplotype determination and population origin We evaluated genetic markers flanking the variant by haplotype analysis in six of the unrelated homozygous patients. We analyzed 14 Single Nucleotide Polymorphism (SNP) and 3 Short Tandem Repeats (STR) by PCR-RFLP and sequencing as described in the Supplementary Materials and Methods.

For mtDNA analysis, we amplified and sequenced a $1016 \mathrm{bp}$ fragment corresponding to the mtDNA control region (rCRS positions 16032-16544 and 051-555) following amplification conditions described previously. ${ }^{16}$

\section{DNA genomic sequencing}

Sanger sequencing of the entire SMPD1 gene of the six homozygous NPDB patients was carried out in the Cologne Center for Genomics (Cologne, Germany) after PCR amplification of a $14.5 \mathrm{~Kb}$ DNA fragment as detailed in the Supplementary Materials and Methods.

Sequences were aligned to the human genome reference GRCh37.p5 using the bwasw tool ${ }^{17}$ for long-read support and split alignment. To correct for Phred-called gap extension sequencing errors, we used the genotype likelihood option. ${ }^{18}$ To compare the haplotype frequencies to data available from the 1000 Genomes Project Phase I, we analyzed the region between positions 6406735 and 6421134 in chromosome 11, which included the SMPD1 gene. We compared unrelated individuals from the following populations: European (CEU; $n=82$ ); African (AFR; $n=88$ ); Chinese (CHB; $n=97$ ) and Mexican samples from Los Angeles (MXL; $n=60$ ). In addition, the results were compared with data obtained from whole genome sequencing in a HuillicheMapuche (HUI; $n=11$ ) cohort, belonging to the Chilean native population (Gutierrez and Miquel (manuscript in preparation)). For the eight SNPs covering the SMPD1 gene all of the possible haplotypes were computed and estimated by multiplying the published frequency of the markers for each population. Significant differences were assessed by the $\chi^{2}$-test.

\section{Vector construction and site-directed mutagenesis}

The p.(Ala359Asp) variant was introduced into the previously described wildtype, full-length SMPD1 cDNA in the pcDNA 3.1(-) expression vector ${ }^{15}$ by PCR-based site-directed mutagenesis using the QuickChange II XL SiteDirected Mutagenesis kit (Stratagene, La Jolla, CA, USA) according to the manufacturer's instructions. The construct was resequenced to ensure that no spurious variants had been introduced by the mutagenesis procedure.

\section{Cell transfection and ASM activity assay}

COS-7 cells were transfected with the wild-type and p.(Ala359Asp) plasmids as detailed in the Supplementary Materials and Methods.

ASM activity was measured in the transfected cells using BODIPY-conjugated C12 sphingomyelin (cat \#D7711; Molecular Probes, Carlsbad, CA, USA) 
as previously described. ${ }^{19}$ Protein concentrations were determined by the Bradford assay. Transfections were performed at least three times for both wildtype and p.(Ala359Asp) constructs. ASM activity of the negative control was subtracted from the expressed activity and the result was expressed as a percentage wild-type construct activity. Data was expressed as mean $\pm \mathrm{SE}$. Statistically significant differences were assessed by the Student's $t$-test.

\section{Immunoblotting}

Cell extracts $(10 \mu \mathrm{g})$ were resolved by SDS-PAGE as detailed in Supplementary Materials. A polyclonal anti-ASM antibody (1:2000 dilution) donated by Dr Edward Schuchman was used.

\section{RESULTS}

Analyses of the p.(Ala359Asp) variant frequency in the Chilean population and theoretical incidence rate of NPDB in Chile

The samples analyzed were Hispanics from a mixed Chilean/Spaniard origin and are representative of at least $70 \%$ of the Chilean population, from the center and south of Chile and were studied before by Miquel et al. ${ }^{20}$ Analysis of 1921 DNA samples from unaffected individuals by the Taqman allelic discrimination assay yielded an $88.0 \%$ call rate of informative DNA samples (1691). Sixteen out of 1691 (0.95\%) samples were heterozygous for the p.(Ala359Asp) variant. SMPD1 DNA sequencing analysis confirmed all heterozygous samples. Thus, the incidence of the p.(Ala359Asp) variant in the Chilean cohort was estimated at $1 / 105.7$, with carrier status probability of 0.0095 (16/1691). If we consider this observation and that the allele frequency for the p.(Ala359Asp) variant is in Hardy-Weinberg equilibrium (HWE), the theoretical incidence of NPDB in Chile could be $1 / 44690$ $(1 / 105.7 \times 105.7 \times 4)$ or greater. This is a higher incidence than estimated from the 45 Chilean NPDB patients diagnosed thus far.

\section{Clinical phenotype of NPDB caused by the p.(Ala359Asp) SMPD1 gene variant}

The mean age at the diagnosis of NPDB was $5.6 \pm 3.6$ years (range $0.7-14.0$ years; the median was 6 years), and at the time of clinical evaluation was $8.0 \pm 3.4$ years (range 1.6-14.8 years) with a median of 8.5 years. The average follow-up of these patients was 10 years. During this time five $(38.5 \%)$ developed a clinically relevant chronic liver

Table 1 Clinical characteristics of 13 NPDB patients homozygous for the p.(Ala359Asp) SMPD1 variant

\begin{tabular}{lc}
\hline Clinical characteristic & $\%$ \\
\hline Moderate to severe hepatosplenomegaly & 100 \\
Increased hepatic transaminases & 100 \\
Dyslipidemia & 92 \\
Epistaxis & 92 \\
Moderate dyspnea during exercise & 54 \\
Gingival bleeding & 54 \\
Anorexia & 46 \\
Thrombocytopenia & 46 \\
Anemia & 38 \\
Recurrent abdominal pain & 38 \\
Progressive cirrhosis & 38 \\
Frequent respiratory infections & 23 \\
Mild to moderate bone pain & 23 \\
Normal eye fundoscopy & 100 \\
Normal intelligence & 100
\end{tabular}

Abbreviations: NPDB, Niemann-Pick disease type B; SMPD1, sphingomyelin phosphodiesterase 1 .

The mean age at the time of this clinical evaluation was $8.0 \pm 3.2$ years old and the follow-up of the patients was 10 years. disease (cirrhosis); three of them died at 6,14 and 15 years of age and the two other patients were liver transplanted.

The most prevalent clinical features of the NPDB Chilean population (Table 1) were moderate to severe hepatosplenomegaly and recurrent epistaxis affecting 13/13 (100\%) and 12/13 (92.3\%) patients, respectively. All of the patients presented normal cognitive levels, normal psychomotor development and moderate growth retardation. About 7/13 (54\%) of the patients presented moderate dyspnea during exercise.

The typical NPDB lipid profile in all except 1 of the 13 patients was characterized by increased total cholesterol and triglyceride levels and decreased high-density lipoprotein (HDL) cholesterol concentration. Mean total cholesterol was $250 \pm 65.5 \mathrm{mg} / \mathrm{dl}(148-376 \mathrm{mg} / \mathrm{dl})$, total cholesterol/HDL cholesterol relation was $9.7 \pm 2.0$ (5.5-11.6) and triglyceride concentration was $250.1 \pm 96.3 \mathrm{mg} / \mathrm{dl}(122-381 \mathrm{mg} / \mathrm{dl})$.

Liver function profile demonstrated persistent increased alanine and aspartate transaminase levels in all patients, being $4.0 \pm 1.9-$ and $4.3 \pm 1.6$-fold of normal values, respectively. Total bilirubin also was abnormally increased in $5 / 13$ patients $(38.5 \%)$, with levels that ranged from 1.25 to $3.8 \mathrm{mg} / \mathrm{dl}(\mathrm{NV} \leq 1.2)$. Mean prothrombin time was $77.4 \pm 11.1 \%$ of that of healthy controls, with a large reduction ( $<70 \%$ of healthy controls) observed in $2 / 13$ patients $(15.4 \%)$.

\section{Haplotype analysis and population origin of the p.(Ala359Asp) variant}

We analyzed DNA markers (both SNP and STR) in six NPDB patients within a $939 \mathrm{~Kb}$ genomic region including the SMPD1 gene. Figure 1 shows patient haplotypes for 12 chromosomes carrying the p.(Ala359Asp) variant.

Notably, all of the p.(Ala359Asp) chromosomes shared a common haplotype between the rs11040859 and rs11040930 SNPs, a region which includes the SMPD1 gene. Patient 3 disrupted the homozygosity and diverged from the other patients in the rs497681 marker, leaving a run of homozygosity that is shared by NPDB patients over a region of at least $280-350 \mathrm{~Kb}$ on chromosome 11 . These results suggest that the variant likely originated from a common founder. Based on the length of the shared haplotype region of $280 \mathrm{~Kb}$, a conservative estimate suggests that a common ancestor carrying the p.(Ala359Asp) variant lived 40 to 60 generations ago (ie, $1200-800$ A.D.). ${ }^{21}$

To determine the population origin of the variant, we calculated the frequency of the shared NPDB patient's haplotype in different populations. We used the haplotypes defined by the eight SNPs in the SMPD1 gene region, which were analyzed by RFLP and confirmed by sequencing. The frequency of each marker was obtained from the 1000 Genomes Project Phase I and from our own data for 11 individuals from a HUI population. The haplotype frequency for each population showed that it is most frequent in HUI and $\mathrm{CHB}$ populations, and less frequent in MXL and CEU populations (Figure 2). This haplotype is found with lowest frequency in the AFR population.

Interestingly, mtDNA analysis revealed that all NPDB patients shared a maternal southern cone Amerindian lineage. ${ }^{22}$ The haplogroup of patient 1 was D1g; patients 2, 4 and 5 had the C1b13 haplogroup; and patients 3 and 6 had the B2i2 and C1b haplogroups, respectively. Haplogroups D1g, B2i2 and $\mathrm{C} 1 \mathrm{~b} 13$ were recently described and are very frequent lineages in populations of the south central region of Chile and southern Argentina. ${ }^{16,23,24}$ These haplogroups are common in the Chilean Huilliche-Mapuche native population, corroborating the results obtained in the frequency haplotype analysis above. 


\begin{tabular}{|c|c|c|c|c|c|c|c|c|c|c|c|c|c|}
\hline \multirow[t]{2}{*}{ DNA marker } & \multirow{2}{*}{$\begin{array}{c}\text { Distance from SMPD1 } \\
\text { or gene region }\end{array}$} & \multicolumn{2}{|c|}{ P1 } & \multicolumn{2}{|c|}{ P2 } & \multicolumn{2}{|c|}{ P3 } & \multicolumn{2}{|c|}{ P4 } & \multicolumn{2}{|c|}{ P5 } & \multicolumn{2}{|c|}{ P6 } \\
\hline & & H1 & $\mathrm{H} 2$ & H1 & $\mathrm{H} 2$ & H1 & $\mathrm{H} 2$ & H1 & $\mathrm{H} 2$ & H1 & $\mathrm{H} 2$ & H1 & $\mathrm{H} 2$ \\
\hline D11S1323 & 5'-upstream (135Kb) & 2 & 4 & 2 & 2 & 4 & 4 & 4 & 4 & 2 & 4 & 2 & 4 \\
\hline D11S4531 & $99 \mathrm{~Kb}$ & 2 & 3 & 2 & 3 & 2 & 3 & 2 & 3 & 2 & 3 & 2 & 3 \\
\hline rs11040859 & $75 \mathrm{~Kb}$ & $\mathrm{~T}$ & $\mathrm{~T}$ & $\mathrm{~T}$ & $\mathrm{~T}$ & $\mathrm{~T}$ & $\mathrm{~T}$ & $\mathrm{~T}$ & $\mathrm{~T}$ & $\mathrm{~T}$ & $\mathrm{~T}$ & $\mathrm{~T}$ & $\mathrm{~T}$ \\
\hline rs2947030 & $68 \mathrm{~Kb}$ & $A$ & $A$ & $A$ & $A$ & $A$ & $A$ & $A$ & $A$ & $A$ & $A$ & $A$ & $A$ \\
\hline rs7128818 & $41 \mathrm{~Kb}$ & C & C & C & C & C & $\mathrm{C}$ & C & C & C & C & C & C \\
\hline rs7940008 & $2.3 \mathrm{~Kb}$ & $\mathrm{C}$ & $\mathrm{C}$ & $\mathrm{C}$ & $\mathrm{C}$ & $\mathrm{C}$ & $\mathrm{C}$ & $\mathrm{C}$ & $\mathrm{C}$ & C & C & C & $\mathrm{C}$ \\
\hline rs1542705 & $1.4 \mathrm{~Kb}$ & $A$ & $A$ & $A$ & $A$ & $A$ & $A$ & $A$ & $A$ & $A$ & $A$ & $A$ & $A$ \\
\hline p.(Ala359Asp) & Exon 2 & $A$ & $A$ & $A$ & $A$ & $A$ & $A$ & $A$ & $A$ & $A$ & $A$ & $A$ & $A$ \\
\hline rs72896270 & Intron 2 & G & G & G & G & G & G & G & G & G & $G$ & G & G \\
\hline rs74053351 & Intron 5 & C & C & C & C & C & C & C & C & C & C & C & C \\
\hline rs11601088 & Intron 5 & C & C & C & C & C & C & C & C & C & C & C & C \\
\hline rs1050239 & Exon 6 & G & G & G & G & G & G & G & G & G & G & G & G \\
\hline rs1800606 & $5.1 \mathrm{~Kb}$ & $\mathrm{~T}$ & $\mathrm{~T}$ & $\mathrm{~T}$ & $\mathrm{~T}$ & $\mathrm{~T}$ & $\mathrm{~T}$ & $\mathrm{~T}$ & $\mathrm{~T}$ & $\mathrm{~T}$ & $\mathrm{~T}$ & $\mathrm{~T}$ & $\mathrm{~T}$ \\
\hline rs2682092 & $6.0 \mathrm{~Kb}$ & $A$ & $A$ & $A$ & $A$ & $A$ & $A$ & $A$ & $A$ & $A$ & $A$ & $A$ & $A$ \\
\hline rs2723663 & $72 \mathrm{~Kb}$ & C & C & C & C & C & C & C & C & C & C & C & C \\
\hline rs11040930 & $200 \mathrm{~Kb}$ & $A$ & $A$ & $A$ & $A$ & $A$ & $A$ & $A$ & $A$ & $A$ & $A$ & $A$ & $A$ \\
\hline rs497681 & $404 \mathrm{~Kb}$ & $\mathrm{C}$ & C & $\mathrm{C}$ & C & C & $\mathrm{T}$ & C & C & $\mathrm{C}$ & C & $\mathrm{C}$ & $\mathrm{C}$ \\
\hline D11S1331 & 3'-downstream (800 Kb) & 5 & 7 & 5 & 7 & 7 & 3 & 1 & 7 & 5 & 7 & 7 & 7 \\
\hline
\end{tabular}

Figure 1 Haplotypes of the six homozygous p.(Ala359Asp) NPDB Chilean patients. The colors indicate the common haplotypes for each patient's chromosomes: pink for p.(Ala359Asp) variant; green for common haplotypes and yellow for non conserved markers. $\mathrm{H} 1$ and $\mathrm{H} 2$ refer to the constructed haplotypes for each patient.

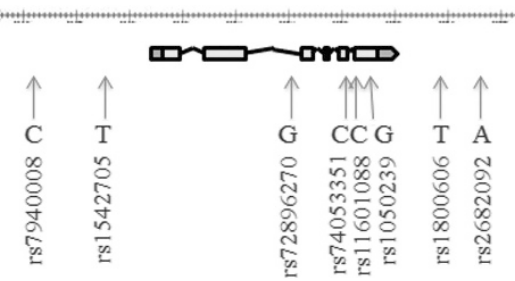

\begin{tabular}{|c|c|c|c|c|}
\hline HUI & CHB & MXL & CEU & AFR \\
\hline $\begin{array}{c}51.78 \% \\
\mathrm{e}\end{array}$ & $\begin{array}{c}43.19 \% \\
\mathrm{c}, \mathrm{d}, \mathrm{e}\end{array}$ & $\begin{array}{c}30.07 \% \\
\mathrm{~b}, \mathrm{e}\end{array}$ & $\begin{array}{c}18.64 \% \\
\mathrm{~b}, \mathrm{e}\end{array}$ & $\begin{array}{c}1.57 \% \\
\mathrm{a}, \mathrm{b}, \mathrm{c}, \mathrm{d}\end{array}$ \\
\hline
\end{tabular}

Figure 2 Population origin of the SMPD1 p.(Ala359Asp) variant. Graphical depictions of the patients' haplotypes constructed from eight SNPs within the SMPD1 gene region, with haplotype frequencies derived from the 1000 Genomes Project: African (AFR; $n=88$ ); Han Chinese in Beijing, China (CHB; $n=97$ ); Utah residents with Northern and Western European ancestry from the CEPH collection (CEU; $n=82$ ); Mexican ancestry from Los Angeles, USA (MXL; $n=60$ ); and from our data (HUI; $n=11$ ). aSignificantly different to HUI; ${ }^{b}$ Significantly different to $\mathrm{CHB}$; ${ }^{\mathrm{c} S i g n i f i c a n t l y ~ d i f f e r e n t ~ t o ~}$ MXL; dSignificantlyy different to CEU; esignificantly different to AFR. $P<0.05, \chi^{2}$-test. SMPD1, sphingomyelin phosphodiesterase 1 ; SNP, single nucleotide polymorphism.

Expression analysis of the p.(Ala359Asp) variant

DNA sequence analysis of the six homozygous p.(Ala359Asp) patients ruled out the presence of other possible causal SMPD1 variants. No other non-coding or coding pathogenic variants in this region were found, confirming that the p.(Ala359Asp) is highly likely to be responsible for the reduced ASM activity in these patients.

To confirm that the p.(Ala359Asp) change affects ASM activity, we used site-directed mutagenesis and transient transfection assays in 

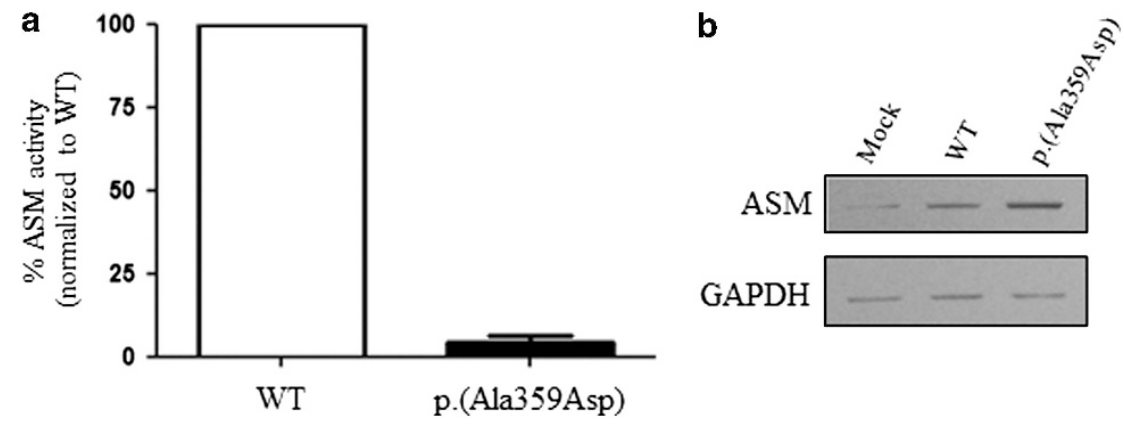

Figure 3 Functional characterization of the SMPD1 p.(Ala359Asp) variant. CoS-7 cells transiently expressing the ASM wild-type (WT) and the p.(Ala359Asp) variants were generated and an ASM activity assay was performed on cell lysates (a). Data represents the mean \pm SE of three to five experiments as a percent of the ASM activity induced by wild-type ASM. ${ }^{*} P<0.0001$. (b) The levels of expression of endogenous, WT and p.(Ala359Asp) ASM protein in cells were assessed by Western immunoblotting. About $10 \mu \mathrm{g}$ of total cell lysate protein was loaded into each lane. ASM, acid sphingomyelinase; SMPD1, sphingomyelin phosphodiesterase 1.

COS-7 cells. The ASM activity in cells expressing the ASM-p. (Ala359Asp) variant allele was significantly lower $(4.2 \pm 1.3 \%)$ than cells expressing the wild-type allele $(1.89 \pm 0.94$ and $59.92 \pm 33.3 \mathrm{U} / \mathrm{mg}$ protein in cells expressing the variant and the wild-type allele, respectively; $P<0.0001$; Figure $3 \mathrm{a}$ ), confirming that this variant has a functional effect in vitro. Importantly, the ASM protein levels were comparable in the wild-type and p.(Ala359Asp) transfected cell lines, as shown in the Western blot analysis in Figure 3b. This indicates that the reduced activity was likely due to an effect on catalytic activity, rather than protein stability or expression.

\section{DISCUSSION}

In this study, we describe the frequency and genetic characterization of the p.(Ala359Asp) variant causing NPDB. Our results show that this variant is frequent in the Chilean population and has a common founder, and that homozygosity leads to moderate to severe NPDB disease. Expression analysis demonstrates significantly reduced ASM activity with relatively normal levels of protein, revealing that the effect of this variant was primarily on catalytic function.

If we consider that the allele frequency for the p.(Ala359Asp) variant is in HWE in the Chilean population, the theoretical incidence of NPDB in Chile would be at least 1/44690. However it should be considered that random mating and thus HWE may not apply in this native population. Despite this, the incidence is higher than the estimated by the number of previously communicated and confirmed clinical cases. In fact, the probability could be even greater, because this calculation only considers the probability of occurrence of the disease by p.(Ala359Asp) homozygosity. Indeed, we also have diagnosed NPDB compound heterozygous Chilean patients.

NPDB occurs at high frequencies in other ethnic groups as well due to specific variants for each population, but no general population studies have been reported yet. ${ }^{8,14,25}$ To our knowledge this is the first detailed epidemiological report on NPDB in any country and the most extensive analysis of a patient population that exhibits homozygosity for a SMPD1 variant. Data such as this has important implications for the implementation of new therapies, including enzyme replacement therapy, and should be considered by the National Health Systems. Appropriate public policies should be developed to reduce the time of diagnosis by supporting healthcare professionals who are unfamiliar with NPDB. Currently, the disease is commonly undetected or misdiagnosed due to its highly variable clinical presentation characterized by a wide range of symptoms that individually are not specific.
The results of this investigation revealed that the p.(Ala359Asp) SMPD1 variant is associated with a moderate to severe NPDB phenotype. The most relevant characteristics are the presence of hepatosplenomegaly; a moderate dyslipidemia; mild to moderate increment of transaminases, mild anemia and thrombocytopenia and moderate to severe pulmonary compromise. However, it should be noticed that the patients have some phenotypic variability, eg, not all of them have respiratory compromise. Indeed, $\sim 40 \%$ of the patients with this variant developed cirrhosis according to our observations. It is intriguing that cirrhosis due to unidentifiable causes (cryptogenic) seems to be frequent in the Chilean population. The high incidence of the p.(Ala359Asp) variant in non-NPD individuals could be a genetic factor to explain a higher susceptibility to cirrhosis in Chileans. This hypothesis will be a topic of future investigations.

Interestingly, our data indicates that the p.(Ala359Asp) variant originates from a common ancestor. This conclusion is based on a common shared haplotype flanking the SMPD1 gene found in homozygosity in all patients' chromosomes. A conservative estimation of the variant age suggests that the common ancestor for the p.(Ala359Asp) variant lived more than 40-60 generations ago (ie, before 1200-800 A.D.). However, it should be noticed that the small number of alleles studied and the recent time frame are likely to result in wide confidence intervals of our estimates.

A Native American origin is therefore most likely responsible for the p.(Ala359Asp) variant since it is estimated that the peopling of Chile with the 'first Americans'26 began $\sim 12000$ years before our era and the Spanish came in contact with the Chilean natives only in the year 1536. Our comparative population analysis of the patient haplotype frequencies also supports the hypothesis of a Native American origin of the variant. Indeed, the highest patient haplotype frequency was found in the HUI population, followed by the closest ethnic groups $\mathrm{CHB}$ and MXL. In contrast, the haplotype was less frequent in patients of the CEU and AFR populations. Although no significant differences were found when comparing the haplotype frequency between the HUI and other populations, with the exception of AFR population, this was likely due to the small number of HUI individuals included in this study $(n=11)$. In contrast, the haplotype frequency in the CHB population was significantly different compared with all other populations $(P<0.05)$, with the exception of the HUI population.

The observed allele frequency could be explained by American settlement from Asia through the Bering Strait, with one group migrating directly to the South of the continent. ${ }^{26}$ Finally, the fact that the six homozygous p.(Ala359Asp) patients studied carried a maternal 
native southern corn lineage mtDNA is also consistent with a Native American origin of the variant. ${ }^{22,24}$

The specific, causal effect of the p.(Ala359Asp) variant on ASM function has not been studied previously. According to bioinformatic modeling of the ASM protein, alanine 359 forms part of, or is located near, the substrate-binding region of this hydrolase. ${ }^{15,27}$ Indeed, this amino acid is highly conserved between diverse species such as human, mouse and worm. ${ }^{27}$ In addition, the alanine to aspartic acid amino acid change, harbored by the p.(Ala359Asp) protein, is predicted to be damaging by several bioinformatics programs. This is consistent with the site-directed mutagenesis/expression evidence reported here, confirming that the p.(Ala359Asp) variant significantly reduces the ASM activity by $>95 \%$ compared with the wild-type enzyme. The clinical phenotype of the homozygous p.(Ala359Asp) patients, consistent with a moderate to severe form of NPDB disease, also suggests that this variant has a serious impact on ASM function.

In conclusion, the present study describes the frequency of the p.(Ala359Asp) SMPD1 gene variant in the general healthy Chilean population and provides an in-depth characterization of the clinical and genomic phenotypes displayed by homozygous patients. Our results show that this variant is frequent in the Chilean population and has a common ancient founder, most probably originated in pre-Columbian Native American population. We also demonstrate that p.(Ala359Asp) is a disease-causing variant by showing reduced ASM activity in cells transfected with the p.(Ala359Asp) cDNA. Additional work is required to establish further biochemical consequences of this variant and to understand the specific disease phenotypes of these patients.

\section{CONFLICT OF INTEREST}

EHS is an inventor on several patents licensed to Genzyme, a Sanofi Corporation, that describes the diagnosis and treatment of NPD. $\mathrm{P}$ Mabe has received a speaker honorarium form Genzyme, a Sanofi Corporation, and BioMarin. Also she has received support for assistance to scientific meetings from Genzyme, BioMarin and Shire. P Mabe and SZ have received research grants from Genzyme, a Sanofi Corporation. The remaining authors declare no conflict of interest.

\section{ACKNOWLEDGEMENTS}

We thank Fermin Robledo for excellent technical support. We also acknowledge the Humboldt Foundation (Germany) for donating the ABI 7500 sequence detection system. This work was supported by grants from the Fondo Nacional de Desarrollo Científico y Tecnológico (FONDECYT; grant numbers 1110310 and 1150186) to SZ and (grant number 1130303) to JFM; Fondo Nacional de Desarrollo de Areas Prioritarias, FONDAP Center for Genome Regulation (CGR; Project number 15090007) to SZ, JFM, MG and RAG; Genzyme grant 'Assessment of the p.(Ala359Asp) acid sphingomyelinase gene variant frequency in the Chilean population' to PM, EHS, SZ; Comisión Nacional de Ciencia y Tecnología (CONICYT) PhD student (grant number 21120490) to MA; National Institutes of Health grant HD28607 to EHS.

1 Schuchman EH, Levran O, Pereira LV, Desnick RJ: Structural organization and complete nucleotide sequence of the gene encoding human acid sphingomyelinase (SMPD1). Genomics 1992; 12: 197-205.

2 Schuchman $\mathrm{EH}$ : The pathogenesis and treatment of acid sphingomyelinase-deficient Niemann-Pick disease. J Inherit Metab Dis 2007; 30: 654-663.
3 Schuchman EH, Desnick RJ: Niemann-pick disease types A and B: acid sphingomyelinase deficiencies; in Valle D (ed): The Online Metabolic and Molecular Bases of Inherited Disease, Online edn. New York, NY, USA: McGraw-Hill Companies, Inc. p 144.

4 Smith EL, Schuchman EH: The unexpected role of acid sphingomyelinase in cell death and the pathophysiology of common diseases. FASEB J 2008; 22 : 3419-3431.

5 Wasserstein MP, Desnick RJ, Schuchman EH et al: The natural history of type B Niemann-Pick disease: results from a 10-year longitudinal study. Pediatrics 2004, 114: e672-e677.

6 Wasserstein MP, Aron A, Brodie SE, Simonaro C, Desnick RJ, McGovern MM: Acid sphingomyelinase deficiency: prevalence and characterization of an intermediate phenotype of Niemann-Pick disease. J Pediatr 2006; 149: 554-559.

7 Pavlu-Pereira H, Asfaw B, Poupctova $\mathrm{H}$ et al: Acid sphingomyelinase deficiency. Phenotype variability with prevalence of intermediate phenotype in a series of twentyfive Czech and Slovak patients. A multi-approach study. J Inherit Metab Dis 2005; 28: 203-227.

8 Simonaro CM, Desnick RJ, McGovern MM, Wasserstein MP, Schuchman EH The demographics and distribution of type B Niemann-Pick disease: novel mutations lead to new genotype/phenotype correlations. Am J Hum Genet 2002; 71 $1413-1419$.

9 McGovern MM, Lippa N, Bagiella E, Schuchman EH, Desnick RJ, Wasserstein MP: Morbidity and mortality in type B Niemann-Pick disease. Genet Med 2013; 15 618-623.

10 Desnick RJ, Schuchman EH: Enzyme replacement therapy for lysosomal diseases: lessons from 20 years of experience and remaining challenges. Ann Rev Genom Hum Genet 2012; 13: 307-335.

11 Platt FM, Lachmann RH: Treating lysosomal storage disorders: current practice and future prospects. Biochim Biophy Acta 2009; 1793: 737-745.

12 Schuchman EH, Simonaro CM: The genetics of sphingolipid hydrolases and sphingolipid storage diseases. Handb Exp Pharmacol 2013; 215: 3-32.

13 Desnick JP, Kim J, He X, Wasserstein MP, Simonaro CM, Schuchman EH: Identification and characterization of eight novel SMPD1 mutations causing types A and B Niemann-Pick disease. Mol Med 2010; 16: 316-321.

14 Mihaylova V, Hantke J, Sinigerska I et al: Highly variable neural involvement in sphingomyelinase-deficient Niemann-Pick disease caused by an ancestral Gypsy mutation. Brain 2007; 130: 1050-1061.

15 Jones I, He X, Katouzian F, Darroch PI, Schuchman EH: Characterization of common SMPD1 mutations causing types A and B Niemann-Pick disease and generation of mutation-specific mouse models. Mol Genet Metab 2008; 95: 152-162.

16 de Saint Pierre M, Bravi CM, Motti JM et al: An alternative model for the early peopling of southern South America revealed by analyses of three mitochondrial DNA haplogroups. PloS One 2012; 7: e43486.

$17 \mathrm{Li} \mathrm{H}$, Durbin R: Fast and accurate long-read alignment with Burrows-Wheeler transform. Bioinformatics 2010; 26: 589-595.

$18 \mathrm{Li} \mathrm{H}$, Handsaker B, Wysoker A et al: The sequence alignment/map format and SAMtools. Bioinformatics 2009; 25: 2078-2079.

19 He X, Chen F, Dagan A, Gatt S, Schuchman EH: A fluorescence-based, highperformance liquid chromatographic assay to determine acid sphingomyelinase activity and diagnose types A and B Niemann-Pick disease. Anal Biochem 2003; 314: $116-120$

20 Miquel JF, Covarrubias C, Villaroel L et al: Genetic epidemiology of cholesterol cholelithiasis among Chilean Hispanics, Amerindians, and Maoris. Gastroenterology 1998; 115: 937-946.

21 Hakonen AH, Davidzon G, Salemi R et al: Abundance of the POLG disease mutations in Europe, Australia, New Zealand, and the United States explained by single ancient European founders. Eur J Hum Genet 2007; 15: 779-783.

22 Moraga ML, Rocco P. Miquel JF et al: Mitochondrial DNA polymorphisms in Chilean aboriginal populations: implications for the peopling of the southern cone of the continent. Am J Phys Anthropol 2000; 113: 19-29.

23 Bodner M, Perego UA, Huber G et al: Rapid coastal spread of First Americans: novel insights from South America's Southern Cone mitochondrial genomes. Genome Res 2012; 22: 811-820.

24 de Saint Pierre M, Gandini F, Perego UA et al: Arrival of Paleo-Indians to the southern cone of South America: new clues from mitogenomes. PloS One 2012; 7: e51311.

25 Vanier MT, Ferlinz K, Rousson R et al: Deletion of arginine (608) in acid sphingomyelinase is the prevalent mutation among Niemann-Pick disease type B patients from northern Africa. Hum Genet 1993; 92: 325-330.

26 Reich D, Patterson N, Campbell D et al: Reconstructing Native American population history. Nature 2012; 488: 370-374.

27 Seto M, Whitlow M, McCarrick MA et al: A model of the acid sphingomyelinase phosphoesterase domain based on its remote structural homolog purple acid phosphatase. Protein Sci 2004; 13: 3172-3186.

Supplementary Information accompanies this paper on European Journal of Human Genetics website (http://www.nature.com/ejhg) 